Supporting Information

\title{
Tumor Associated Neutrophil Extracellular Traps Regulating Nanocarrier Enhanced Inhibition of Malignant Tumor Growth and Distant Metastasis
}

Haoyuan Yin ${ }^{a, c \S}$, Hongdan Lu ${ }^{a \S}$, Yaokun Xiong ${ }^{d \S}, L u Y^{a}{ }^{a}$, Chuanhui Teng ${ }^{a}$, Xiang Cao ${ }^{a}$, Shengnan Li $i^{a}$, Shanbo Sun ${ }^{a}$, Wentao Liu ${ }^{e}$, Wei Lv $v^{b *}$, Hongliang Xin ${ }^{a *}$

${ }^{a}$ Department of Pharmaceutics, School of Pharmacy, Nanjing Medical University, Nanjing 211166, China

${ }^{b}$ Department of Pharmacy, the Affiliated Jiangyin Hospital of Southeast University Medical College, Jiangyin 214400, China

c Institute of Dermatology, Chinese Academy of Medical Sciences and Peking Union Medical College, Nanjing 210042, China.

${ }^{d}$ Department of Pharmaceutics, School of Pharmacy, Jiangxi University of Traditional Chinese Medicine, Nanchang 330004, China

${ }^{\mathrm{e}}$ Department of Pharmacology, School of Basic Medicine, Nanjing Medical University, Nanjing 211166, China

*E-mail: 1vwei_1201@163.com; xhl@njmu.edu.cn

$\S$ These authors contributed equally 


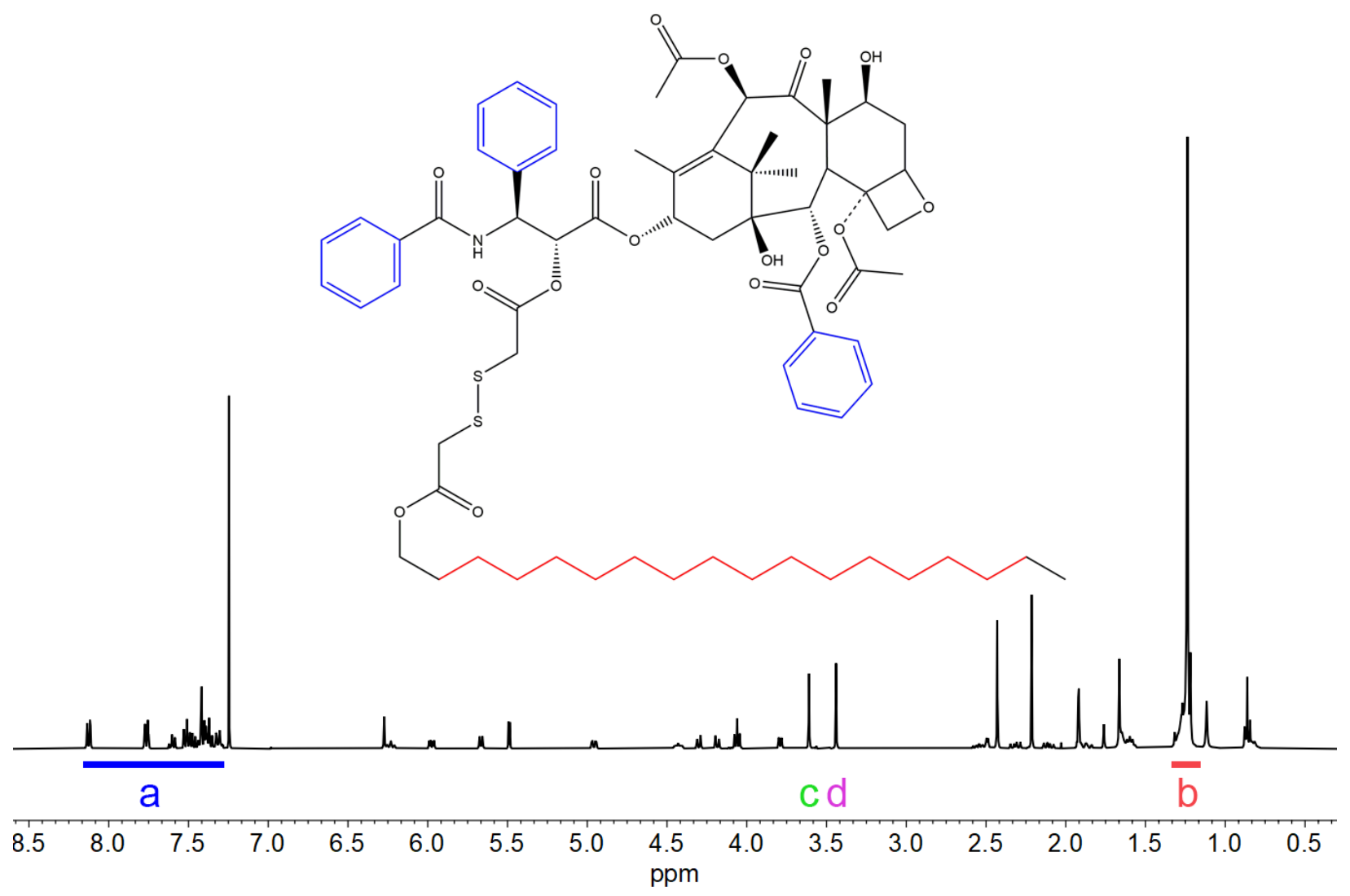

Figure S1. ${ }^{1} \mathrm{H}$ NMR spectrum of PTX-SS-C18. (a) The proton peak of the H atom on the three benzene rings. (b) The proton peak of $\mathrm{H}$ atom in methylene group of octadecyl. (c, d) The proton peaks of $\mathrm{H}$ atoms on two methylene groups connected by disulfide bonds 


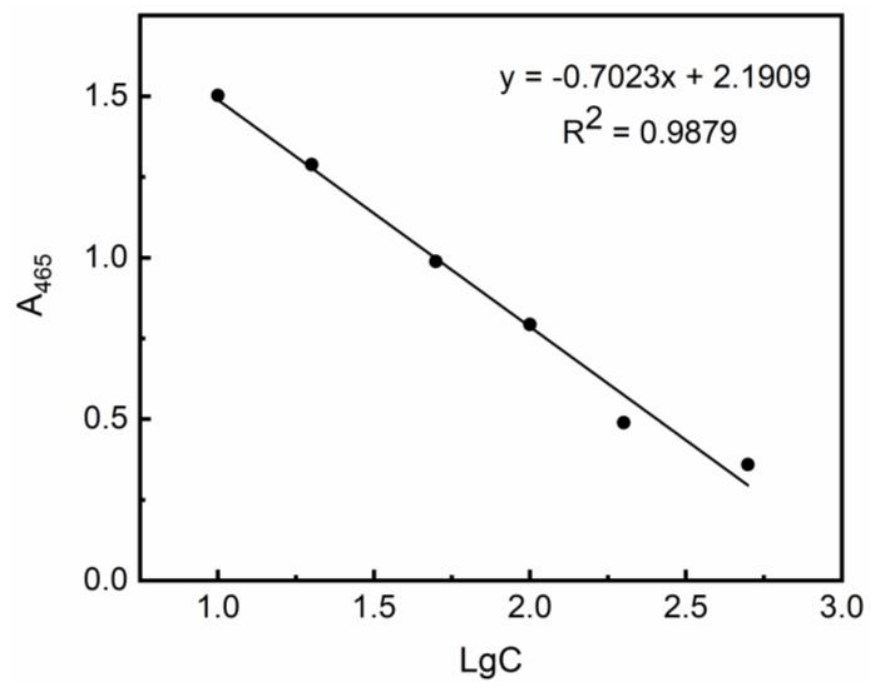

Figure S2. Variation of A464 of supernatants with methyl orange concentration. 

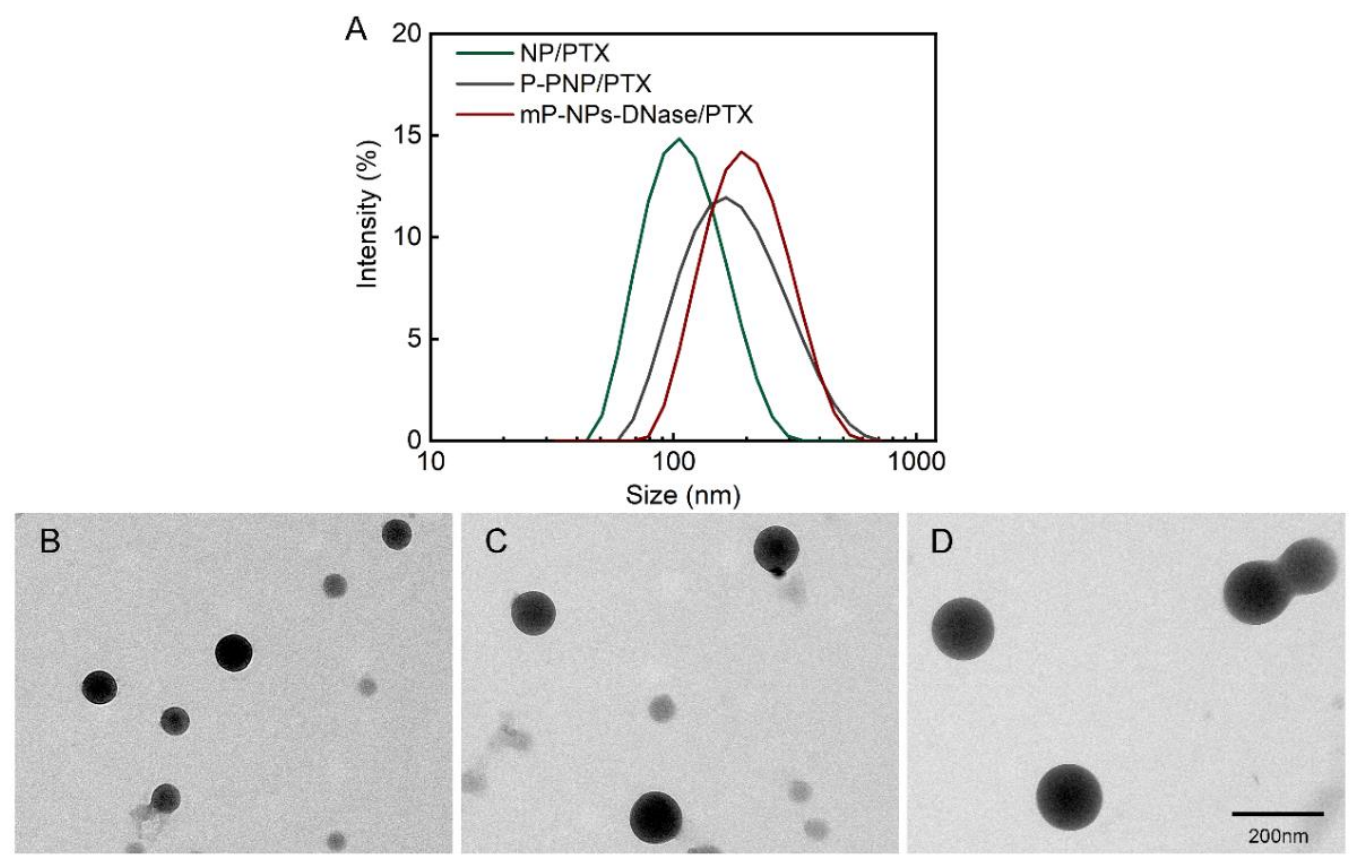

Figure S3. Characterization of nanoparticles. (A) Hydrodynamic size distributions of NP/PTX, PPNP/PTX and mP-NPs-DNase/PTX. Typical TEM images of NP/PTX (B), P-PNP/PTX (C) and mP-NPs-DNase/PTX (D). Scale bar: $200 \mathrm{~nm}$. 


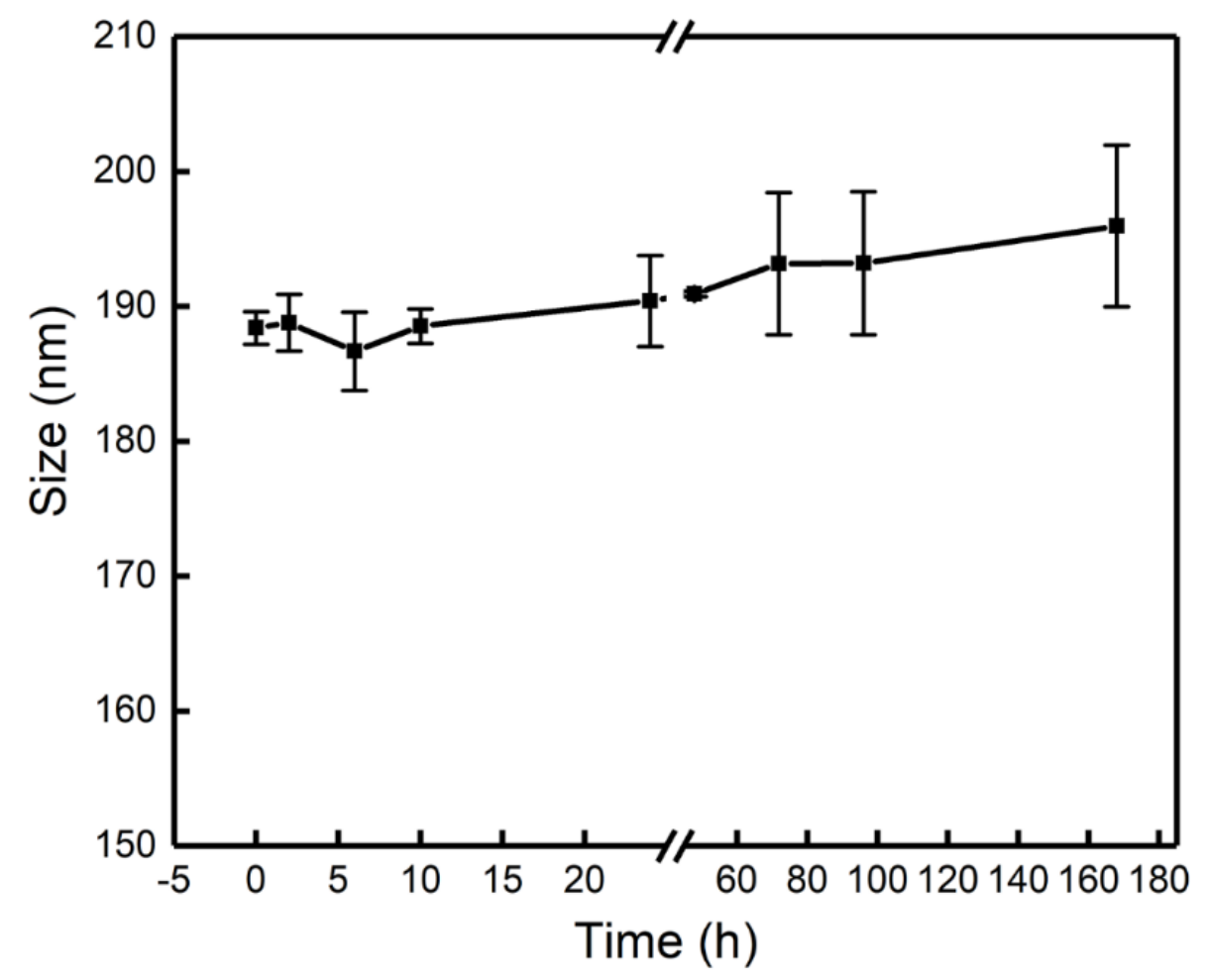

Figure S4. The hydrodynamic diameters of mP-NPs-DNase/PTX in PBS at $4{ }^{\circ} \mathrm{C}$ for 7 days. Data represent mean $\pm \mathrm{SD}(\mathrm{n}=3)$. 


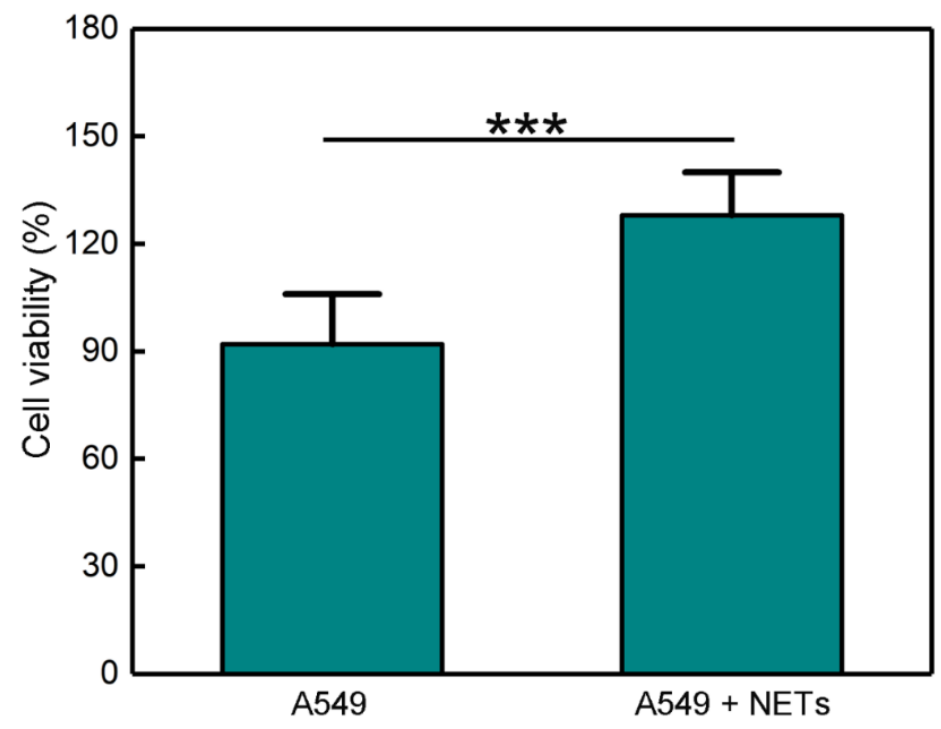

Figure S5. Positive effect of NETs on proliferation of A549 cells. Data represent mean $\pm \operatorname{SD}(n=5)$. Significant differences between the indicated groups: $* * * P<0.001$. 


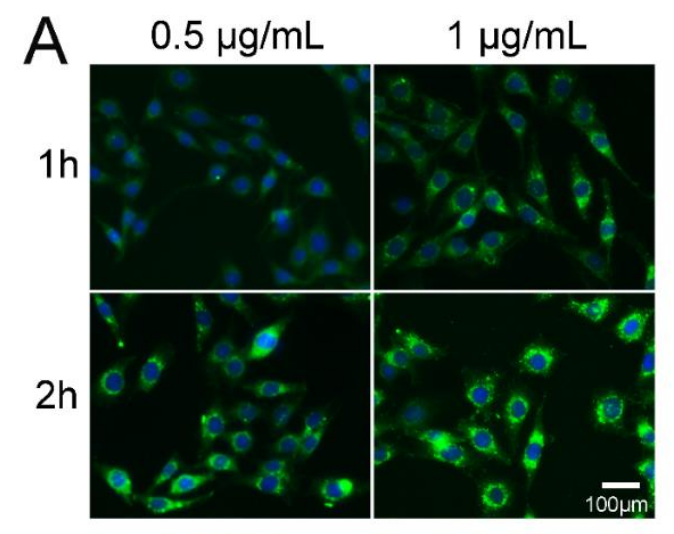

Hoechst 33342 Coumarin - 6

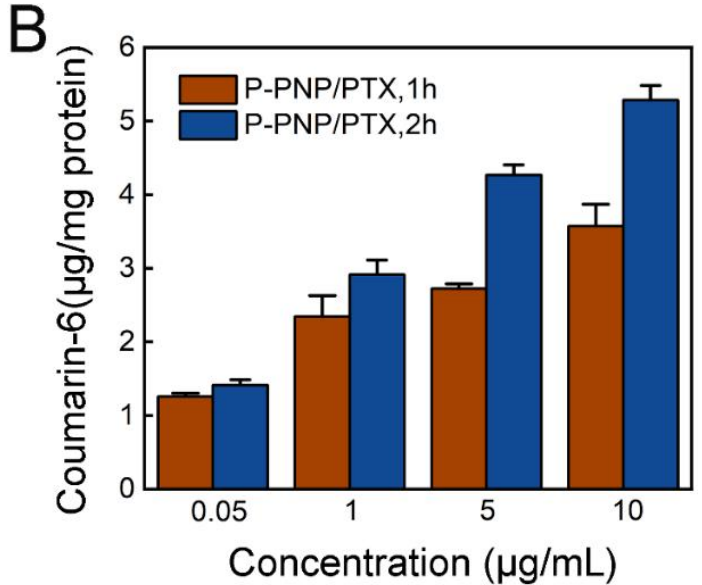

Figure S6. Cellular uptake. (A) The fluorescence qualitative uptake of coumarin-6 labeled PPNP/PTX by A549 for $1 \mathrm{~h}$ or $2 \mathrm{~h}$. Scale bar: $100 \mu \mathrm{m}$. (B) The fluorescence quantitative uptake of coumarin-6 labeled P-PNP/PTX by A549 for $1 \mathrm{~h}$ or $2 \mathrm{~h}$. Data represent mean \pm SD $(n=4)$. 

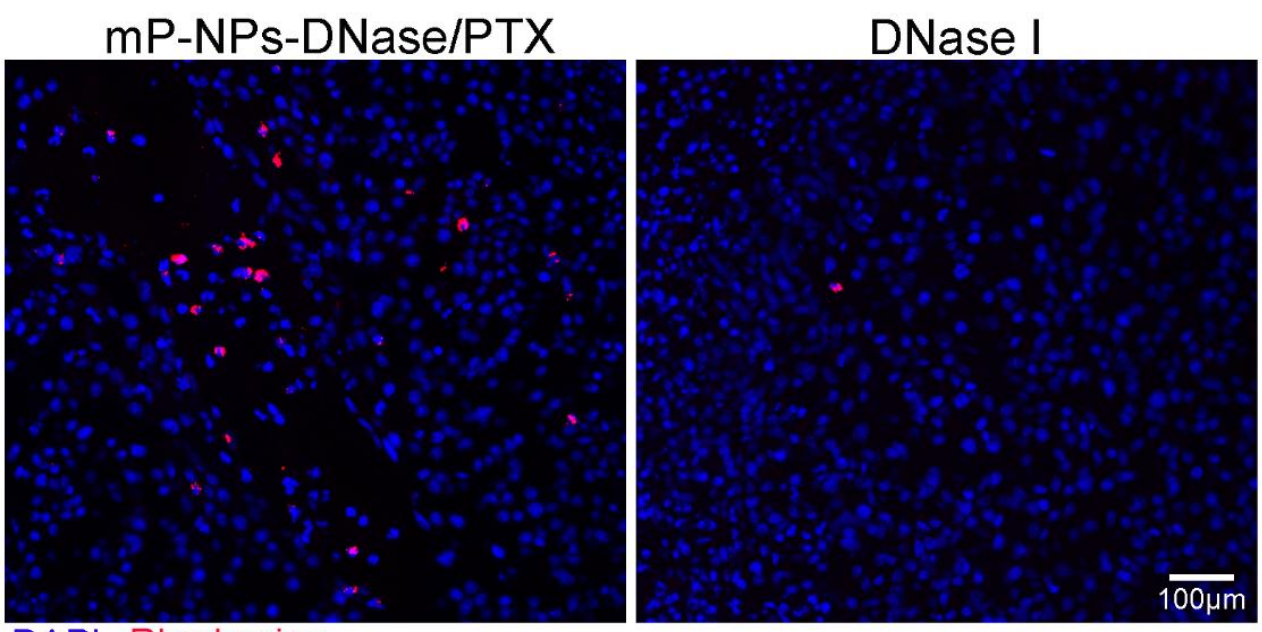

DAPI Rhodamine

Figure S7. Accumulation of DNase I grafted on mP-NPs-DNase/PTX and free DNase I in tumor site. Scale bar: $100 \mu \mathrm{m}$. 


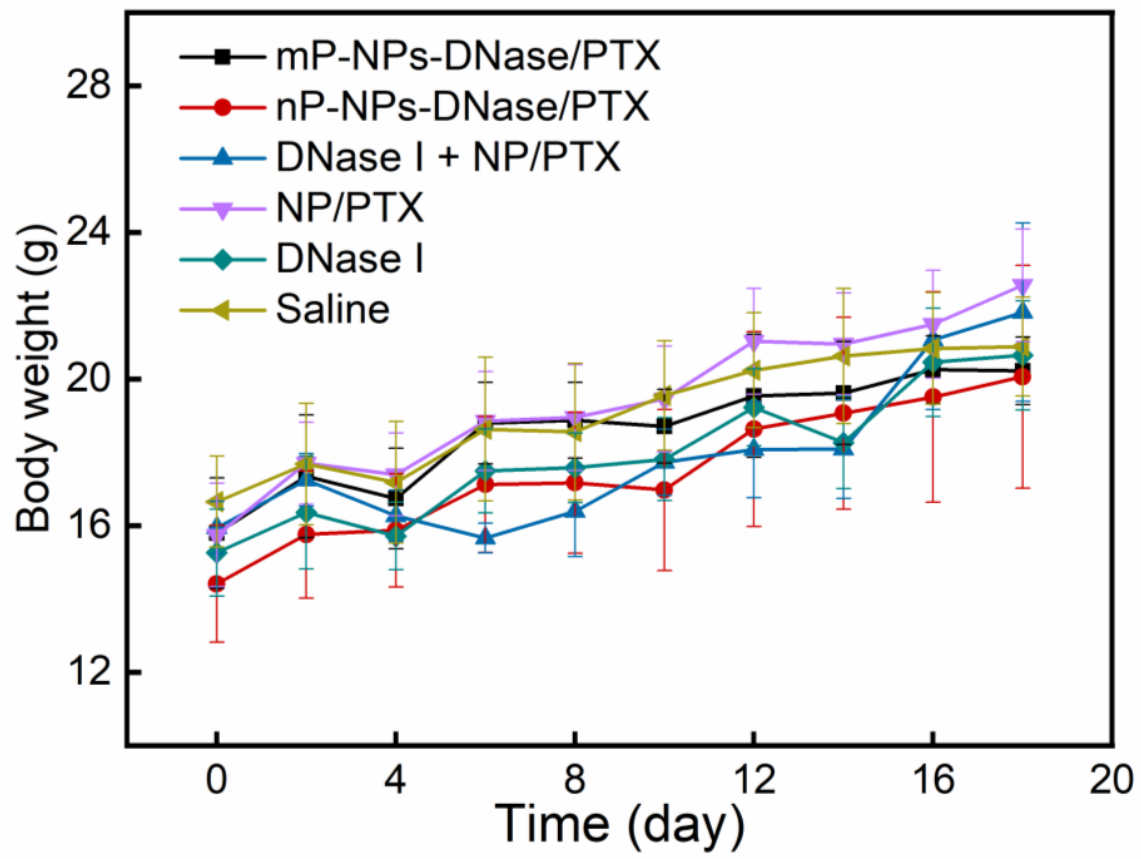

Figure S8. Weight changes of nude mice bearing tumor. Data represent mean \pm SD $(n=6)$. 


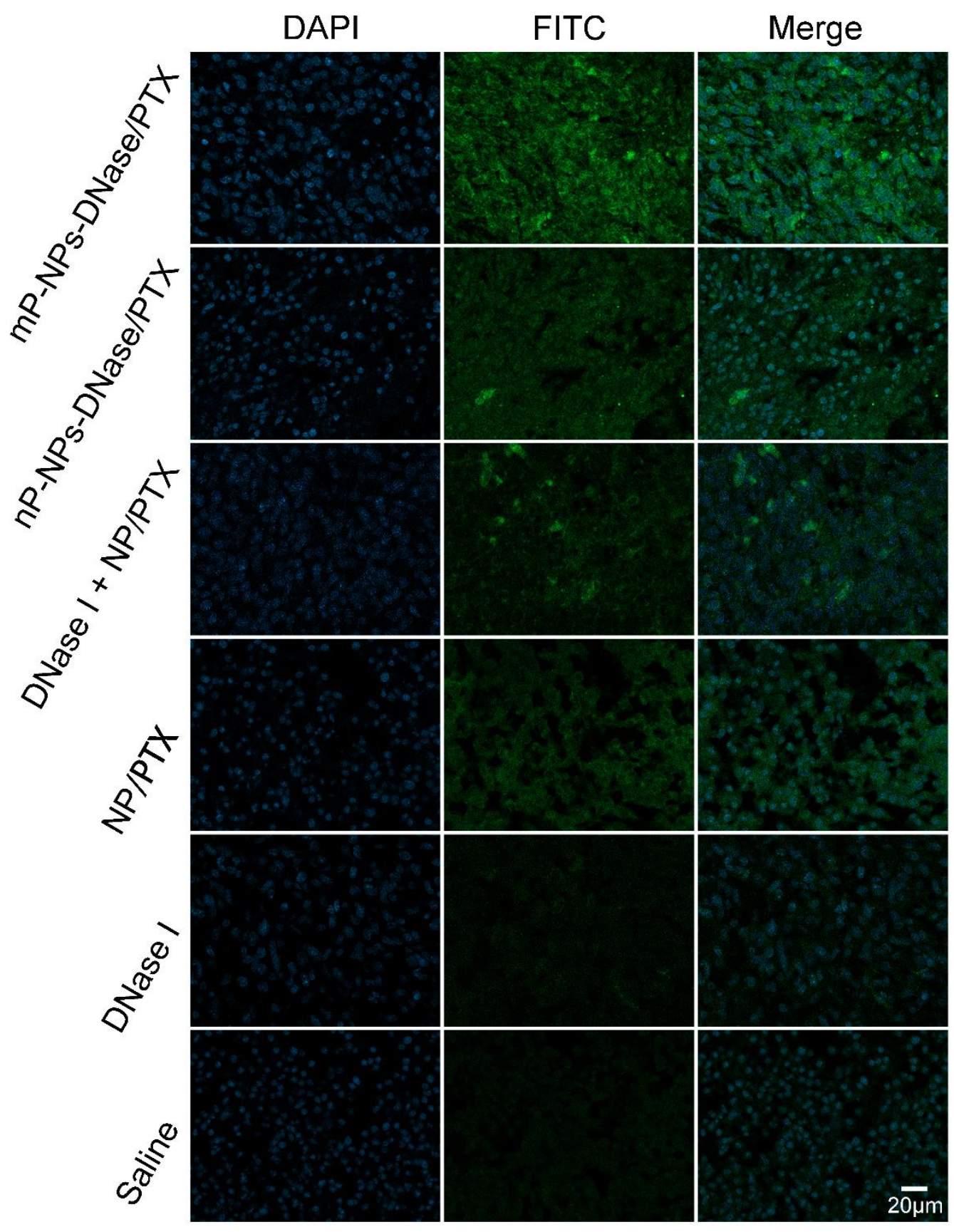

Figure S9. Tumor apoptosis of different administration groups. Scale bar: $20 \mu \mathrm{m}$. 


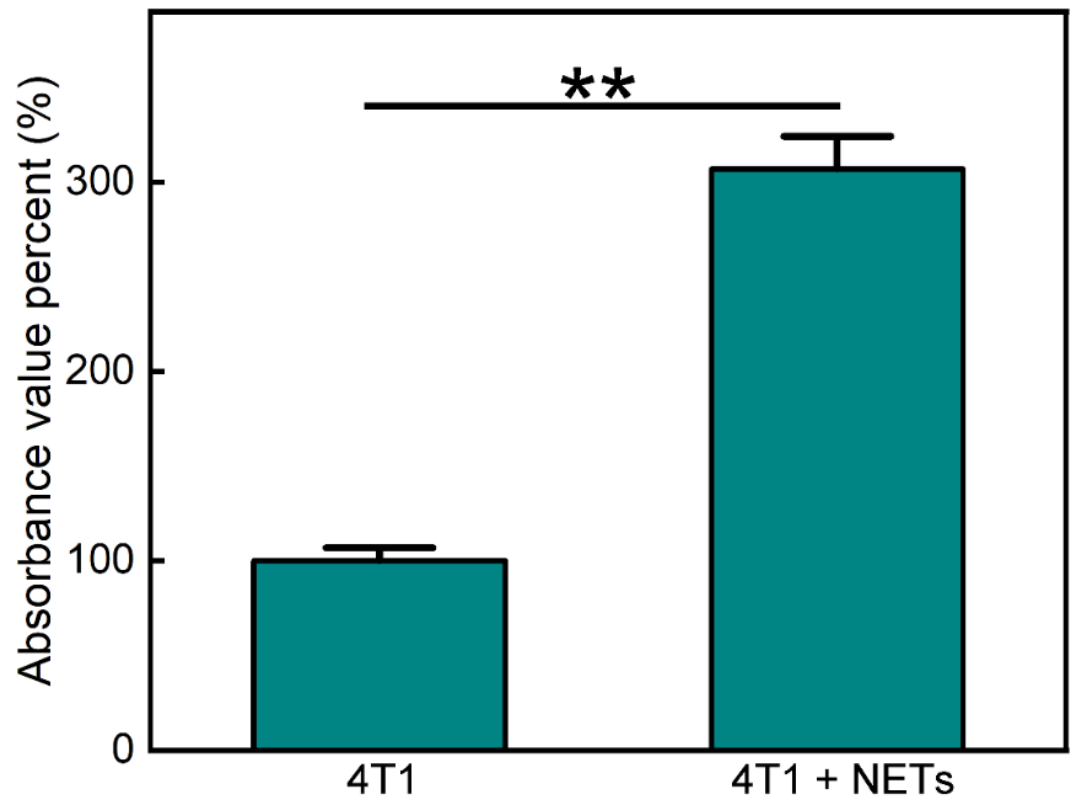

Figure S10. Effects of NETs on migration of $4 \mathrm{~T} 1$ cells. Data represent mean \pm SD $(n=6)$. Significant differences between the indicated groups: $* * P<0.01$. 

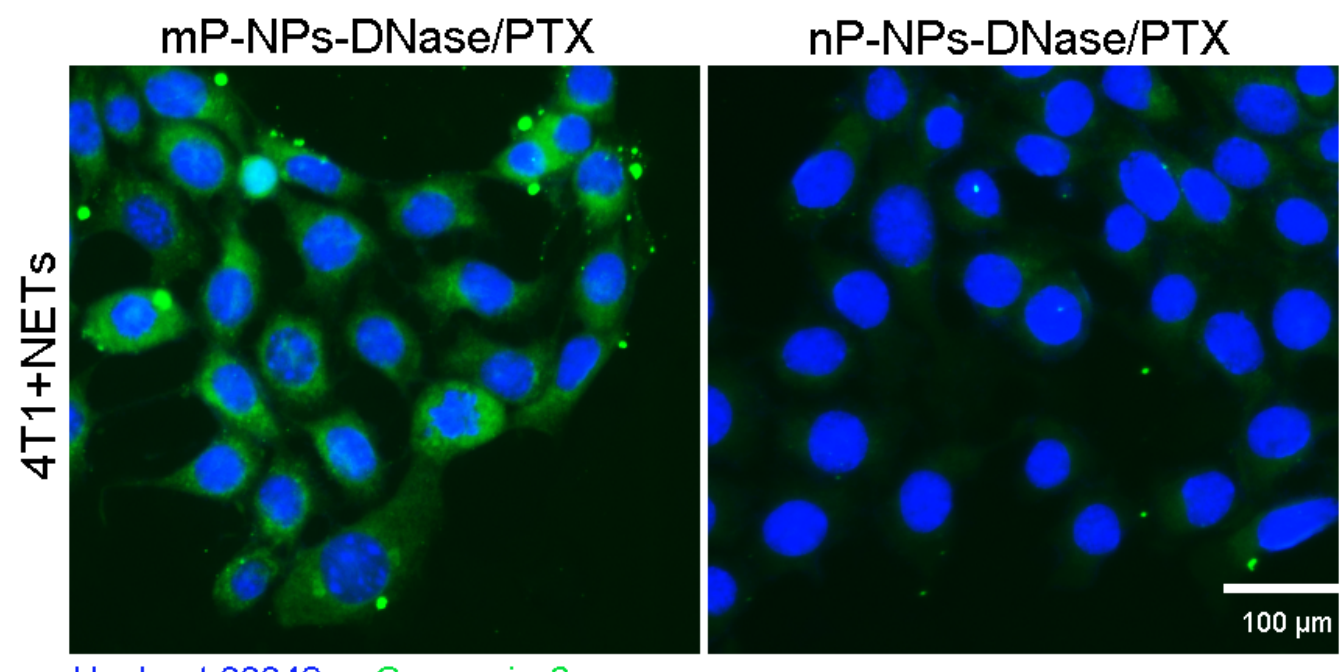

Hochest 33342 Coumarin-6

Figure S11. Qualitative uptake of mP-NPs-DNase/PTX and nP-NPs-DNase/PTX in 4T1 cells cocultured with NETs. Scale bar: $100 \mu \mathrm{m}$. 
Table S1. Characterization of NP/PTX, P-NP/PTX and mP-NPs-DNase/PTX.

\begin{tabular}{cccc}
\hline Formulation & Particle Size & Polydispersity & Zeta Potential \\
& $(\mathrm{nm})$ & Index $(\mathrm{PDI})$ & $(\mathrm{mv})$ \\
\hline NP/PTX & $106.1 \pm 0.53$ & $0.113 \pm 0.026$ & $-27.83 \pm 0.59$ \\
P-NP/PTX & $149.37 \pm 1.59$ & $0.116 \pm 0.016$ & $24.37 \pm 0.81$ \\
$\mathrm{mP-NPs-DNase/PTX}$ & $187.27 \pm 0.67$ & $0.168 \pm 0.005$ & $-11.97 \pm 0.25$ \\
\hline
\end{tabular}

